\title{
ASPECTS OF CONTRACEPTION AND REPRODUCTIVE HEALTH IN OBESE ADOLESCENT GIRLS
}

\author{
Laura-Mihaela Trandafir'1, Madalina Ionela Chiriac ${ }^{2}$, Oana Teslaru ${ }^{3}$, \\ Ingrith Miron ${ }^{4}$, Oana Chirila ${ }^{5}$, Smaranda Diaconescu $^{6}$ \\ ${ }^{1} I I I^{r d}$ Department of Pediatrics, "St. Mary” Emergency Hospital for Children, \\ "Grigore T. Popa" University of Medicine and Pharmacy, Iasi \\ ${ }^{2}$ Endocrinology Department, Clinical County Emergency Hospital, Cluj-Napoca \\ ${ }^{3}$ Parodontology Department of "Grigore T. Popa" University of Medicine and \\ Pharmacy, Iasi \\ ${ }^{4} I V^{\text {th }}$ Department of Pediatrics, "St. Mary" Emergency Hospital for Children, \\ "Grigore T. Popa" University of Medicine and Pharmacy, Iasi \\ ${ }^{5}$ Department of Pediatrics, "St. Mary" Emergency Hospital for Children, Iasi \\ ${ }^{6} V^{\text {th }}$ Department of Pediatrics, "St. Mary” Emergency Hospital for Children, \\ "Grigore T. Popa” University of Medicine and Pharmacy, Iasi
}

\begin{abstract}
Unintended pregnancies are a serious problem in terms of morbidity during the adolescent years, the use of contraceptives being an important element of prevention at this age.

Obese adolescent girls tend to use fewer contraceptive methods compared to normal-weight girls, excess weight being a frequent argument against the use of contraception.

These girls have a high probability of carrying unintended pregnancies associated with all the risks of pregnancy and childbirth at very young ages, while also needing safer and more effective contraceptive methods. Reducing the number of pregnancies in this category is a public health priority and requires the identification of adolescent girls included in this risk category. Although there are several voices opposing contraceptive methods on account of their adverse effects, the World Health Organization champions the idea that adolescents can use any contraceptive method due to the benefit of avoided unintended pregnancies, which outbalance the risks associated with the use thereof.
\end{abstract}

Keywords: adolescent girls, obesity, pregnancy, contraception

\section{INTRODUCTION}

Obesity at the age of adolescence reached an epidemic level, with current data showing that over the last 30 years the rate of obese teenagers worldwide quadrupled. In the United States of America, the rate of obesity in the 12-19 years of age group increased from 5\% in 1980 to $21 \%$ in $2012(1,2)$. According to the Health Report of Children and Youth in Romania (2014) regarding the causes of illness in the 2012-2013 academic year, non-endocrine obesity ranked second after refractive errors (5.04\%), followed by the pathology of the vertebral column (1.9\%), ponderal hypotrophy (1.71\%) and sequelae of rickets $(1.58 \%)$. According to the same report, 1 in 3 children aged 6 to 9 years old are overweight or obese. The incidence of overweight (including obesity) in children aged 11 to 13 years old ranges from $5 \%$ to over $25 \%$. (3) Adolescents with excessive weight or obesity are confronted with serious health problems from a medical, psychological, as well as reproductive point of view. Early dietary intervention is essential for preventing obesity-related comorbidities from occurring both in the short and long run. Adolescence is a transitional period comprising physical, behavioral and personality changes. Adolescents become interested in their own appearance and their position 
compared to their peers. Obesity at this age has serious consequences on shaping the autonomy, identity and sexual adjustment of the future adult. In addition to the multiple organic complications (cardiovascular, endocrine, gastrointestinal, pulmonary and osteoarticular), the psychological complications of obesity are just as important at this age. Depression, low self-esteem and the social bias regarding obesity are challenging factors that make it difficult for obese adolescents to manage their weight. Therefore, the family, school and society must identify and support adolescents with nutritional problems. Adolescents depend on their collaboration with their family, teachers, the school psychologist and physician in order to tackle dietary counselling issues, as well as issues regarding sex life and contraceptive methods, prevention of sexually transmitted diseases and drug consumption.

\section{Obesity associated with pregnancy and psycho-emotional disorders occurring in adolescent girls}

The data available in the specialty literature shows that over $60 \%$ of the children who are overweight before puberty will grow up to become overweight adults, which will lead to the development of chronic illnesses such as cardiovascular disease and type II diabetes. $25 \%$ of the 15 year olds have had sexual intercourse and in some countries over $30 \%$ of them do not use condoms or any other form of contraception, which leads to sexually transmitted diseases and unintended pregnancies in adolescent girls (3). Unintended pregnancies are a serious problem in terms of morbidity during the adolescent years, the use of contraceptives being an important element of prevention at this age $(4,5)$. The risk of death in adolescent mothers is four times higher in girls under 16 as compared to women over the age of 20 . Up to $65 \%$ of the women presenting with obstetric complications had a history of pregnancy during adolescence (6). Pregnancy during adolescence is an undesirable situation that puts the young expectant mother in a special set of circumstances and exposes her to risks that adult expectant mothers experience less frequently. The causes of unintended pregnancies in adolescent girls include precarious socio-economic conditions, low level of education, early marriages, especially in the case of particular ethnic and religious groups, precocious and unprotected sexual intercourse, older partners, abuse, rape, consumption of alcohol and/or drugs, peer pressure and the influence exerted by mass media. Pregnancies during adolescence encompass a higher risk of complications, such as stillbirth, premature delivery, premature birth, low birthweight, neonatal asphyxia, newborn deaths occurring in the first week of the first month of life (7). The main obstetric complication in adolescent girls is premature birth, especially if the time of conception occurred shortly after the menarche (8). The psycho-emotional problems determined by pregnancy during adolescence have been reported as early as 1980. Faigel reported psycho-emotional effects on the children of adolescent mothers; 20 men and women that became parents during adolescence were invited to a follow-up interview 17 to 24 years later. All the pregnancies that had occurred during adolescence were first seen as a situation of dissonance or failure to establish a parent-child relationship due to their rebellious adolescent behavior and implicitly that of the child born during their own adolescence. Children born to adolescent parents had an adequate behavior during childhood, but after becoming adolescents and having age-specific activities (such as going out), parents deemed the latter negative and even risky in terms of repeating the parents' "mistake". Adolescent mothers turned out to be openly spying on their daughters, expressing their mistrust regarding their heterosexual relationships. All the parents were unable to discuss sexuality-related matters with their children (9). The inadequate conditions of intrauterine growth, prematurity, birth-related difficulties such as difficult expulsion, low birthweight, pregnancy-related anemia and malformations have been described along with delayed psychosomatic development, behavioral and language disorders that can occur in the child, as well as other long term complications. Some studies associate pregnancies during adolescence with a predominantly negative evolution, being attributable to certain socio-demographic factors such as poverty, low level of education, inadequate prenatal care and unmarried (marital) status $(10,11)$. According to the "Adolescent Pregnancy Guidelines" of the Society of Obstetricians and Gynecologists in Canada, there was an annual worldwide record of 14 million births in underage mothers, aged between 14 and 19 years of age (12).

\section{Contraception and its effective use in obese adolescent girls}

The WHO champions the idea that that adolescents can use any contraceptive method and that they should have access to a variety of contracep- 
tive methods. Age is not an impediment in any of the contraceptive options. Although there are opinions opposing oral contraceptives due to their side effects (13) (nausea, weight gain, dysmenorrhea, and sometimes the aggravation of acne), their advantages should be considered in order to avoid unintended pregnancies at very young ages (6). The WHO recommends various birth control methods for adolescent girls, while also trying to eliminate the financial barriers to the use of contraceptives and to improve access to information about contraceptives (14). Contraception in adolescent mothers is necessary due to their significant risk of becoming pregnant again, a risk that is $25 \%$ higher during the next 2 years after giving birth. The prevention of repeated pregnancies in adolescent girls during the postpartum period can be made using long-acting reversible contraception such as DMPA (depot medroxyprogesterone acetate) and intrauterine devices, which have a higher success rate than short term contraceptive methods such as oral contraceptives, contraceptive patch or barrier methods (diaphragm). The long-acting reversible contraceptive methods are particularly approved for adolescent girls by the American Congress of Obstetricians and Gynecologists. Contraception administered immediately post-partum with post-partum DMPA initiation, IUD insertion after delivery or insertion of a contraceptive implant before hospital release helped to significantly reduce the rate of repeat pregnancies (12). A study carried out by Thurman et al. showed that $14.2 \%$ of the adolescent girls that used DMPA became pregnant one year later, as compared to $29.7 \%$ and $31.8 \%$ of adolescent girls that used oral and patch-type contraceptive methods (15). The use of secure and effective contraception is essential for preventing unintended pregnancies, particularly among adolescents. Several aspects have to be considered when counselling obese adolescent girls about contraception: the manner in which excess weight impacts the security and effectiveness of various contraception methods as well as the effects of contraceptive on body weight (16-18). A longitudinal study carried out by Chang et al. among adolescent girls aged 18 to 19 years old regarding sexual behavior showed that obese girls were less likely to use contraceptive methods and less likely to use them consistently, as compared to the normal-weight girls. Thus, the reported data suggest that obesity can be considered an important factor associated with the sexual behavior of overweight/obese adolescent girls (19). According to the guidelines for the use of contraceptives for adolescent girls in the USA, the health and sexual behavior related requirements of obese adolescents are substantially assimilated with those of normal-weight people. As far as the approach to contraception is concerned, it is important to note that obesity and its related endocrine effects can influence the effectiveness and side-effects of contraceptives. For instance, a low number of pregnancies occurring within a short period of time were reported among users of transdermal patch-type contraception with a body weight of over $90 \mathrm{~kg}$. The report of the World Health Organization and the Centers for Disease Control and Prevention shows that the available data on the effectiveness of combined oral contraceptives (COC) is limited and inconsistent. Moreover, this data varies depending on body weight or body mass index (BMI). It was reported that obese women that use $\mathrm{COC}$, vaginal ring or intrauterine devices present with no changes in terms of weight pursuant to COC initiation compared to normal-weight women. However, it was noted that obese adolescent girls that use DMPA are more likely to gain weight than those who do not use DMPA, but the body weight increases both in obese adolescent girls that use COC and in normal-weight girls that use DMPA. An increasing number of adolescents are undergoing bariatric surgery and they need effective contraceptive methods. The available data reveals a post-surgical improvement of fertility, which is likely to reduce the effectiveness of contraceptives due to the malabsorption syndrome, vomiting and diarrhea. It is recommended to delay pregnancies for at least 12 months after the bariatric surgical procedure (20). It was discovered that the majority of a small population of adolescents that presented to the hospital with venous thromboembolism (VTE) associated with contraception also presented with multiple VTE risk factors. The study included 26 young female patients aged 12 to 21 years old, who had been admitted for VTE related with hormonal contraception. Of the 57 reviewed cases of VTE, 26 were found to be connected to contraception. $96 \%$ of the patients had at least one additional VTE risk factor and $42 \%$ of the patients had 2 or more such risks factors. $50 \%$ of the patients had a BMI $\geq 25$ $\mathrm{kg} / \mathrm{m} .35 \%$ of the patients had a positive family history of VTE in their $1^{\text {st }}$ and $2^{\text {nd }}$ degree relatives. $27 \%$ of the patients were subsequently diagnosed with hereditary thrombophilia, of which 5 had a positive family history. Obesity was the most frequent additional risk factor $(50 \%)$ identified in the study population. Further research is necessary regarding the impact of obesity on contraception-related VTE in adolescent girls, and on whether obe- 
sity could influence the screening practices for thrombophilia before prescribing contraceptive methods (21).

\section{Obesity and endocrine disorders occurring in adolescent girls}

Obesity is currently a serious public health problem, therefore making it a public health priority to reduce the number of pregnancies in obese adolescent girls and requiring the identification of adolescent girls in this risk category.

Excess weight is thought to be a cause of reduced efficiency in oral contraceptives at the age of adolescence. The adipose (fat) tissue is considered a genuine endocrine organ. Excess fat affects fertility by increasing the risk of irregular menstrual cycles and polycystic ovary syndrome (22). Visceral obesity is responsible for the high levels of androgens that also affect the ovarian function (23). Obesity-associated insulin-resistance is responsible for ovulatory dysfunctions and the decreased levels of sex hormone binding globulin (SHBG). SHGB is a plasma protein involved in the specific binding of androgen hormones and circulating estradiol. It is synthesized in the liver, and its secretion is controlled via a feed-back mechanism by the circulating levels of androgens and estradiol. The concentration of SHBG influences both the tissular bioavailability of sexual steroids, and the estrogenandrogen balance. High levels of androgens inhibit SHBG synthesis, while high levels of estradiol stimulate it. Thus, in patients with polycystic ovary syndrome, obesity and hypothyroidism, SHBG are low. However, it is important to note that most of the obese adolescent girls will continue to ovulate regularly. Although ovulation does not occur every month, there is a high risk of unintended pregnancy (24).

\section{DISCUSSIONS}

Studies show that obesity can be an important risk factor in association with the sexual behavior of adolescents. Women that become pregnant as early as their adolescence have a higher risk of becoming obese later in life. Complications such as gestational diabetes, arterial hypertension, hypercoagulability, and stillbirth occur more frequently in obese pregnant adolescent girls. Also, due to the neonatal pathology, their newborn babies need to be admitted into neonatal intensive care units, as compared to neonates carried by mothers with a normal nutritional status. Understanding the sexual behavior in correlation with the weight of the adolescent girls is essential, as these risks involve both the mothers and their babies (25-27). In addition to the medical problems (affecting both the baby and the mother) and the socio-economic problems entailed by pregnancy during adolescence, psychological disorders are another aspect that should not at all be neglected. Pregnant underage girls have feelings of uncertainty and insecurity that generate anxiety, insomnia, intense feelings of ambivalence and confusion, as well as certain symptoms of depression, feelings of sadness, suicidal thoughts and irritability. As a result, somatic disorders, anxiety and insomnia, severe depression, feelings of guilt and low self-esteem will lead to family and social isolation along with school dropout, thus creating a vicious circle that further worsens psychosocial and psychiatric problems. As far as depressive manifestations in adolescent mothers are concerned, it was noticed that adolescent girls aged between 15 and 17 years were twice as depressed as adult mothers, and severe depression symptoms were more frequently encountered in this age group compared to the 19 to 20 years age group $(28,29)$. All the sexually active adolescent girls should be provided with contraceptive counselling. Obese adolescent girls are at a high risk of unintended pregnancies, along with all the risks entailed by very young pregnancy and require a safe and effective contraceptive method (30). It is a known fact that obesity is a risk factor in endometrial hyperplasia and endometrial cancer; hormonal contraception provides protection for the endometrium and is associated with a low incidence of these pathologies (31-34). Birth control pills proved to be safe and effective, especially in the care of adolescent girls. Encouraging obese girls to use oral birth control pills should continue in spite of several failed cases having been reported (35). The specialty literature also shows that adolescents with healthy lifestyles and that use oral hormonal birth control pills, intrauterine devices or other contraceptive methods do not become overweight or obese (17). The literature clearly highlights, particularly in the case of oral hormonal birth control pills, that there is no difference in terms of weight between women that use this type of medication and those who do not (36). Although the use of oral birth control pills does not determine weight gain, their use can trigger certain changes, such as: increase of body fat percentage and decrease of muscle mass percentage (37). A decrease of performances during physical activities could also be noticed after 3 years of continuous use of oral birth control pills, ergo the 
misassociation of the weight gain with the use of contraceptive methods instead of with the lifestyle changes and adoption of a sedentary lifestyle (38). Adverse effects such as the increased risk of venous thrombosis should also be considered when discussing about oral hormonal contraceptive methods. Obesity in itself is associated with a high risk of venous thrombosis and pulmonary embolism in women, particularly during the first year of using oral contraceptives $(39,40)$.

\section{CONCLUSIONS}

Obesity at the age of adolescence is a public health problem with multiple comorbidities.

\section{REFERENCES}

1. Ogden C.L., Carroll M.D., Kit B.K. et al. Prevalence of childhood and adult obesity in the United States, 2011-2012. JAMA. 2014; 311(8):806-14.

2. National Center for Health Statistics. Health, United States, 2011: With Special Features on Socioeconomic Status and Health. Hyattsville, MD; U.S. Department of Health and Human Services. Washington DC, 2012

3. Raport național de sănătate a copiilor şi tinerilor din România 2014 , pag 4. http://insp.gov.ro/sites/cnepss/wp-content/uploads/2014/11/ Raportul-National-a-Copiilor-si-Tinerilor-2014.pdf

4. Kost K., Henshaw S. US Teenage Pregnancies, Births and Abortions 2010: National and State Trends and Trends by Race and Ethnicity. New York: Guttmacher Institute, 2014. https://www.guttmacher.org/ sites/default/files/report_pdf/ustptrends10.pdf

5. Finer L.B., Zolna M.R. Unintended pregnancy in the United States: incidence and disparities. Contraception. 2011; 84(5):478-85.

6. WHO Adolescent Pregnancy Fact Sheet, 2008. http://www.who.int/ mediacentre/factsheets/fs364/en/

7. Elfenbein D.S., Felice M.E. Adolescent Pregnancy. In: Kliegman R.M Nelson Textbook of Pediatrics $19^{\text {th }}$ Edition. Elsevir. United States of America, 2011. Chapter 112:699-702.

8. Treffers P.E. Teenage pregnancy, a worldwide problem. Ned Tijdschr Geneeskd. 2003; 147(47):2320-5.

9. Faigel H.C. Late Social and psychological aftereffect of pregnancy in adolescence. J Adolesc Health Care. 1982; 2(3):209-12.

10. Chen X.K., Wen S.W., Fleming N. et al. Teenage pregnancy and adverse birth outcomes: a large population based retrospective cohort study. Int J Epidemiol. 2007; 36(2):368-373.

11. Larson C.P. Poverty during pregnancy: Its effects on child health outcomes. Paediatr Child Health. 2007; 12(8):673-677.

12. Fleming N., O'Driscoll T., Becker G. et al. Society of Obstetricians and Gynaecologists of Canada. Adolescent Pregnancy Guidelines. J Obstet Gynaecol Can. 2015 Aug; 37(8):740-59.

13. Cromer B. Contraception. In: Kliegman R.M. Nelson Textbook of Pediatrics 19 ${ }^{\text {th }}$ Edition. Elsevir. United States of America, 2011. Chapter 111:692-699.

14. WHO MEC 2009. http://apps.who.int/iris/ bitstream/10665/181468/1/9789241549158_eng.pdf

15. Thurman A.R., Hammond N., Brown H.E. et al. Preventing repeat teen pregnancy: postpartum depot medroxyprogesterone acetate, oral contraceptive pills, or the patch?. J Pediatr Adolesc Gynecol. 2007; 20:61-5.
Advancing public health strategies that are based on the promotion of a healthy lifestyle and regular exercise regimen is a nation-wide priority for the purpose of preventing noncommunicable chronic diseases. Solid knowledge regarding contraceptive methods will help the pediatrician both in promoting a healthy sex life, as well as in solving gynecological problems affecting adolescent girls. Statistics show that well over half of teenagers become sexually active during high-school, most of them having no access to adequate protection against unintended pregnancies and sexually transmitted diseases. In conclusion, it would seem that the multidisciplinary approach (pediatrician, gynecologist, nutritionist and psychologist) is the optimal solution in the case of this category of adolescent girls.

16. WHO, Ensuring Human Rights in the provision of contraceptive information and services. Guidance and recommendations, 2014. http://apps.who.int/iris/bitstream/10665/102539/1/9789241506748_ eng.pdf

17. Wuntakal R., Hollingworth T. The implications of obesity on pregnancy. Obstetrics, Gynecology, and Reproductive Medicine 2009; 19(12):344-359.

18. Whitaker A., Gilliam M. Contraception for Adolescent and Young Adult Women. Springer. 2014:15-23.

19. Chang T., Davis M.M., Kusunoki Y. et al. Sexual Behavior and Contraceptive Use among 18- to 19-Year-Old Adolescent Women by Weight Status: A Longitudinal Analysis. J Pediatr. 2015; 167(3):58692. doi: 10.1016/j.jpeds.2015.05.038. Epub 2015 Jul 1.

20. Committee on Adolescence, Braverman PK, Adelman WP et al. Contraception for adolescents. Pediatrics. 2014;134(4):e1244-56. doi: 10.1542/peds.2014-2299.

21. Pillai P., Bonny A.E, O’Brien S.H. Contraception-related venous thromboembolism in a pediatric institution. J Pediatr Adolesc Gynecol. 2013; 26(3):186-8. doi: 10.1016/j.jpag.2013.02.009. Epub 2013 Apr 6.

22. Hsu Roe A., Dokras A. The Diagnosis of Polycystic Ovary Syndrome in Adolescents. Rev Obstet Gynecol. 2011; 4(2): 45-51.

23. Chuang C.H., Chase G.A., Bensyl D.M., Weisman C.S. Contraceptive use by diabetic and obese women. Womens Health Issues. 2005; 15(4):167-73.

24. Grimes D.A., Shields W.C. Family planning for obese women: challenges and opportunities. Contraception. 2005; 72(1) :1-4.

25. Lopez L.M., Grimes D.A., Chen M., et al. Hormonal contraceptives for contraception in overweight or obese women. Cochrane Database Syst Rev. 2010; 7; (7):CD008452. doi: 10.1002/14651858.CD008452. pub2.

26. Edelman A., Kaneshiro B. Contraception counseling for obese women. UpToDate Available from: http:// www.uptodate.com/contents/ contraception-counseling-for-obesewomen

27. Kaneshiro B., Edelman A. Contraceptive considerations in overweight teens. Curr Opin Obstet Gynecol. 2011; 23:344-9.

28. Deal L.W., Holt V.L. Young maternal age and depressive symptoms: Results from the 1988 national maternal and infant health survey. Am J Public Health. 1998;88(2):266-270.

29. Kaneshiro B., Edelman A., Carlson N. et al. The relationship between body mass index and unintended pregnancy: Results from the 2002 National Survey of Family Growth. Contraception. 2008; $77(4): 234-238$. 
30. Chuang C.H., Chase G.A., Bensyl D.M., Weisman C.S. Contraceptive use by diabetic and obese women. Women's Health Issues. 2005; 15:167-173.

31. Holt V.L., Cushing-Haugen K.L., Kaling J.R. Body weight and risk of oral contraceptive failure. Obstet Gynecol. 2002; 99(51):820-827.

32. Holt V.L., Scholes D., Wicklund K.G. et al. Body mass index, weight, and oral contraceptive failure risk. Obstet Gynecol. 2005; 105:46-52.

33. Brunner Huber L.R., Hogue C.J., Stein A.D. et al. Body mass index and risk for oral contraceptive failure: a case-cohort study in South Carolina. Ann Epidemiol. 2006; 16(8):637-43.

34. ACOG Practice Bulletin. Use of hormonal contraception in women with coexisting medical conditions. Clinical Management Guidelines for Obstetrician-Gynecologists. Obstet Gynecol. 2006;107:1453-72.

35. Greydanus D.E., Omar H.A., Tsitsika A. Concepts of Contraception for Adolescents with Obesity: Pathways of Judicial Moderation. Pediatrics Faculty Publications 2009. Paper 137. http://uknowledge. uky.edu/pediatrics_facpub/137
36. Gallo M.F., Grimes D.A., Schulz K.F., Helmerhorst F.M.

Combination estrogen-progestin contraceptives and body weight: systematic review of randomized controlled trials. Obstet Gynecol 2004; 103(2):359-73.

37. Berenson A.B., Rahman M. Changes in weight, total fat, percent body fat, and central-to-peripheral fat ratio associated with injectable and oral contraceptive use. Am J Obstet Gynecol 2009; 200(3):329. e1-8.

38. Keller C., Larkey L., Distefano J.K. et al. Perimenopausal obesity. J Womens Health (Larchmt). 2010; 19(5):987-96.

39. Trussell J., Guthrie K.A., Schwarz E.B. Much ado about little: obesity, combined hormonal contraceptive use and venous thrombosis. Contraception. 2008; 77(3):143-6.

40. Anton D.K., Kann L., Kinchen S. et al. Centers for Disease Control and Prevention $(C D C)$. Youth risk behavior surveillance - United States, 2011. MMWR Surveill Summ. 2012; 61(4):1-162. 